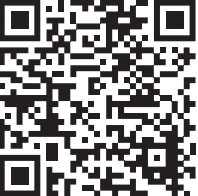

* Médico legista en la Secretaría de Salud de la Ciudad de México.

Correspondencia: IGG, ismaelgarciagarduza@ yahoo.com.mx

Conflicto de intereses: No existe conflicto de intereses en el contenido y publicación de este artículo.

Citar como: García Gl. Mala práctica médica. Caso médico-legal y dictamen. Rev CONAMED. 2021; 26(2): 68-81. https:// dx.doi.org/10.35366/100349 Financiamiento:

Ninguno.

Recibido: 06/10/2020 Aceptado: 26/05/2021.

\section{Mala práctica médica. Caso médico-legal y dictamen}

\author{
Medical malpractice. Medical-legal case and opinion \\ Ismael García Garduza*
}

\section{RESUMEN}

Introducción: Un paciente acude a un médico/hospital solicitando atención médica, esperando que el facultativo utilice su conocimiento y habilidad de la manera más apropiada. El médico debe informar al paciente y familiar sobre el estado del paciente, su manejo y posibles complicaciones que se pueden presentar y si cuenta o no con la especialidad para llevar a cabo su estudio y tratamiento; además de prever que en la unidad médica se cuente con los recursos humanos y la infraestructura para el manejo de complicaciones que aseguren una atención óptima. El objetivo de este artículo es exponer un caso médico-legal y el dictamen realizado por el autor sobre irregularidades en la atención médica y en la integración de los procedimientos de consentimiento informado, expediente clínico y delegación de funciones y las posibles consecuencias profesionales y jurídicas por una denuncia. Conclusión: El desprestigio ocasionado por una mala práctica médica deteriora el ejercicio de esta profesión. Por lo que resulta importante que los estudiantes de medicina y los médicos conozcan casos médico-legales que les sirvan de realimentación para prevenir actos $u$ omisiones que puedan ser determinados por la autoridad como ilegítimos y aprendan a manejar conflictos y resolverlos adecuadamente, y no en la forma en que actuaron los médicos en el caso expuesto en este artículo.

Palabras clave: Mala práctica médica, dictamen médico, expediente clínico, consentimiento informado, delegación de funciones.

\section{ABSTRACT}

Introduction: A patient goes to a doctor/hospital requesting medical attention, hoping that the practitioner will use her knowledge and skill in the most appropriate way; and the doctor must inform the patient and family about the patient's condition, their management and possible complications that may arise and whether or not they have the specialty to carry out their study and treatment; in addition, to foresee that the medical unit has the human resources and infrastructure for the management of complications that ensure optimal care. The objective of this article is: to present a Medico-legal case and the opinion made by the author on irregularities in medical care and in the integration of the procedures of: Informed Consent, Clinical File and Delegation of functions and the possible professional and legal consequences for an accusation. Conclusion: The loss of prestige caused by a medical malpractice deteriorates the exercise of this profession. Therefore, it is important that medical students and physicians know medico-legal cases that serve as feedback to prevent acts or omissions that may be determined by the authority as illegitimate and learn to handle conflicts and resolve them properly and not in the way the doctors acted in the case exposed in this article.

Keywords: Medical malpractice, medical opinion, medical record, informed consent, delegation of functions. 


\section{INTRODUCCIÓN}

La profesión médica se considera noble porque ayuda a preservar la salud y la vida. Una persona enferma generalmente se acerca a un médico u hospital en función de la reputación de éstos. Las expectativas de un paciente son dobles: se espera que tales doctores y hospitales brinden tratamiento medicinal con todo el conocimiento, habilidad y recursos necesarios y, en segundo lugar, que no harán nada que pueda dañarlo de alguna manera, ya sea por negligencia, impericia, actitud imprudente de su personal o falta de recursos.

El galeno lo logrará con un estándar de cuidado, que es un principio de derecho establecido, donde dicho médico aportará a su tarea un grado razonable de habilidad y conocimiento y deberá ejercer un grado razonable de cuidado. Ni el más alto ni el más bajo grado de cuidado y competencia, juzgados a la luz de las circunstancias en cada caso, es lo que exige la ley. El incumplimiento de ese principio da lugar a una acción contra el galeno por mala práctica médica.

Aunque es posible que un facultativo no esté en condiciones de salvar la vida de su paciente, en todo momento se espera que utilice su conocimiento y habilidad especiales de la manera más apropiada, teniendo en cuenta el interés del enfermo que le ha confiado su vida, además de verificar que en el hospital donde se llevará a cabo su intervención estén disponibles los medios que aseguren una atención óptima.

Asimismo, a menos que sea una emergencia, deberá obtener el consentimiento informado del afectado antes de proceder con cualquier tratamiento o intervención, de los establecidos en la Norma Oficial Mexicana NOM-004-SSA3-2012 «Del expediente clínico».

Todo enfermo y sus familiares necesitan una explicación veraz de su padecimiento que les resulte comprensible y convincente y así cooperar en su tratamiento. Esto es cierto, tanto si se trata de dar un diagnóstico en una situación esperanzadora, como de confirmar un mal pronóstico.

Por lo tanto la honestidad del profesional es importante para los afectados. Lo necesitan porque están enfermos, vulnerables y abrumados con preguntas urgentes que requieren respuestas veraces.
No decir la verdad en la relación médico-paciente requiere una atención especial porque los enfermos, hoy más que nunca, sufren graves daños si se les miente; no sólo se socava su autonomía, sino que, a quienes no se les dice la verdad sobre una intervención, experimentan una pérdida de esa importante confianza que se requiere para su curación.

Aquello que los médicos niegan, distorsionan, evaden, fabrican, consideran irrelevante, no responden, omiten, manipulan, revelan u ocultan, como estrategia de engaño, es de gran trascendencia para sus pacientes, porque puede dañar las relaciones y comprometer la atención clínica. Una de las situaciones más graves es cuando se utilizan los expedientes clínicos con ese objetivo.

Los expedientes clínicos forman una parte importante del manejo de un enfermo; es fundamental que los doctores y establecimientos de salud los mantengan integrados e intactos, porque los ayudará en la evaluación científica del perfil del individuo y también a analizar los resultados del tratamiento. Pero de igual importancia en el entorno actual es el tema de la presunta mala práctica médica; el sistema legal se basa principalmente en pruebas documentales en una situación en la que el paciente o los familiares alegan inconformidades con la atención médica proporcionada.

En una queja, la documentación clínica es a menudo la evidencia más importante en la decisión sobre la sentencia o la absolución del médico, en consecuencia, será de gran ayuda para el doctor acusado si documenta claramente que la atención se encuentra dentro del estándar de atención correspondiente. Un archivo incompleto, alterado, o uno que no respalde claramente la atención dentro del estándar establecido, será una desventaja significativa dentro de la defensa del caso.

Por tales motivos, el objetivo de este artículo es revisar los procedimientos de consentimiento informado y expediente clínico, su elaboración de acuerdo con la norma y cuando esa confección es defectuosa e ilegítima por parte del médico; todo ello analizando actos de un facultativo, con los que pretendía engañar para evitar su responsabilidad por los malos resultados en el manejo de una paciente y la forma en que los realizó y cómo se ponen en evidencia por una denuncia penal, que originó el análisis pericial de los hechos y documentos. Todo lo anterior 
se describe a continuación, demostrando la mala práctica médica, con el fin de que estudiantes y médicos traten de aclarar y resolver los conflictos con honestidad y no recurriendo a recursos ilegítimos.

\section{CASO MÉDICO-LEGAL}

\section{Antecedentes}

1. Declaración del denunciante de día 06 de septiembre de 2019.

...mi esposa estaba embarazada y se atendió en Clínica Uno, en todo su embarazo; el día cinco de septiembre del año en curso, a las 10:05 horas ingresó a esa clínica porque el médico que la estaba tratando le dijo que necesitaba una cesárea, y antes de entrar a cirugía le practicaron unos estudios, los cuales señalaban que no tenía problemas para ser intervenida y el cirujano, a las 16:30 horas, ordenó el traslado de mi esposa al quirófano, pasaron alrededor de 20 minutos y la enfermera después de 30 minutos me entrega a mi bebé, le pregunté, qué tal había salido mi esposa, contestándome que va a tardar media hora más, pasaron alrededor de dos horas, por lo que pregunté a la enfermera y me informó que en cuestión de unos 75 minutos más me la entregaban, por eso, siendo las 19:00 horas de la tarde salí a tomar alimentosy a las 20:79 horas, recibo llamada de mi madre, quien me dice que me trasladara al hospital ya que se había puesto mal mi esposa, llego a las 20:35 horas, encontrando en el pasillo del hospital chorros de sangre y no me permitian el acceso a la habitación, hasta que la sacaron en la camilla, desvanecida, y el doctor me dijo que se había complicado, que habían pinchado el útero, que la iban a volver a abrir para checar que todo marchara bien, pasaron 10 minutos y una persona me dijo ya la estabilizamos, necesitamos hacerle una transfusión de sangre y necesitamos bolsas ya que no contamos con ellas, viendo que esta misma persona saca su teléfono y marcaba para ver quién les podía prestar un tanque de oxígeno, ya que no contaban con él, 10 minutos después el doctor me dice, su esposa ya está bien sólo fue una pequeña hemorragia y alrededor de las 00:40 de la mañana vuelve a salir y me dice que era necesario trasladarla porque no contaban con oxígeno y la camilla estaba dañada, y tenía respiración por medio de una bombilla manual; la bajan a la ambulancia para llevarla al Hospital General de México, la ingresan y ahí los médicos nos informan que mi esposa ya no tenía signos vitales, que estaba a punto de entrar en paro y, a las 03:00 horas, me informan que había fallecido.

Asimismo, contrario a lo determinado en el expediente clínico de la parturienta, que establece lo siguiente: 5-09-2019 nota de admisión a urgencias. 16:20 horas paciente femenino 28 años gesta III, cesárea I, partos O, abortos I. Acude tras referir disminución de movimientos fetales desde dos horas previas a acudir a este nosocomio, niega datos de vasoespasmo... paciente femenina 28 años, con embarazo de 38 semanas de gestación por fecha última de men struación (término). Presenta sufrimiento fetal agudo. Por lo que se decide interrupción del embarazo por cesárea por el riesgo de asfixia neonataly muerte del producto in útero.

\section{Al respecto, el denunciante aclara:}

Ingresamos en la Clínica Uno a las 10:15 horas del día cinco de septiembre de 2019, por lo que es falso que hubiera ingresado a urgencias hasta las 16:20 horas del mismo día. Debo resaltar que mi esposa se estuvo tratando con personal de dicha clínica desde el tercer mes de embarazo, y su médico tratante lo fue en todo momento el médico imputado, quien programó como fecha para realizar la cesárea el cinco de septiembre de 2019, a las 10:00 horas, por lo que es falsa la anotación de que en ese momento se decidió la interrupción del embarazo por cesárea. Mi esposa entró a la clínica sin necesitar ayuda, sin presentar sufrimiento fetal agudo y sin malestar alguno, acudimos en virtud de la cirugía programada por el propio médico imputado, refiriendo que serían necesarios estudios preoperatorios, los cuales se practicaron el mismo día, aproximadamente a las 12:00 horas. Es evidente que el expediente clínico fue manipulado, pues los hechos ocurrieron el cinco de septiembre de 2019, y el expediente clínico fue exhibido hasta el 25 de septiembre de 2019.

En los documentos denominados «Conformidad del paciente y familiares para tratamiento 
médico y/o quirúrgico» y "Carta consentimiento bajo información de procedimientos diagnósticos y terapéuticos de alto riesgo», aparecen firmas, a pesar de que el suscrito firma con su nombre.

2. Declaración efectuada por el médico imputado, el 17 de diciembre del 2019:

a. Que ejerzo la profesión de Médico Homeópata Cirujano y Partero.

b. Padecimiento actual: paciente de 28 años de edad que acude por primera vez a este nosocomio, con embarazo de 35.6 semanas por fecha de última menstruación. Gesta III, parto 0, aborto 7 (2018), cesárea 7. Refiere control prenatal irregular, no especifica unidad médica. Refiere polaquiuria y disuria desde hace dos días, acompañado de dolor tipo cólico con irradiación lumbosacra (tipo obstétrico) de dos a tres veces al día de leve intensidad. Refiere salida de flujo blanco amarillento desde hace dos días acompañado de prurito vaginal.

A la exploración física: Pelvimetría no útil. Diámetro conjugado verdadero menor 10 centímetros. Diagnóstico: embarazo de 35.6 semanas de gestación. Primera vez que acude a esta unidad. Infección de vías urinarias. Cervicovaginitis. El día (5) de septiembre de (2019), siendo (16:20 horas), la paciente acude al Servicio de Urgencias de la Clínica Uno, refiriendo disminución de movimientos fetales desde dos horas previas. Niega datos de vasoespasmo. Presenta actividad uterina de 24 horas de evolución, refiere dolor tipo cólico con irradiación lumbosacra, niega pérdidas transvaginales. A la exploración física: tensión arterial de 90/60, frecuencia cardiaca de 80 por minuto, frecuencia respiratoria de 20 por minuto, temperatura de 36.6 grados centígrados, peso de 84.500 kilogramos. Paciente consciente, intranquila, orientada, hidratada, pupilas reactivas. Abdomen, útero gestante, con altura de fondo uterino de 29 centímetros, presentación cefálica, situación longitudinal, dorso derecho, producto libre, frecuencia cardiaca fetal de 92 latidos por minuto. Tacto vaginal con cérvix posterior, largo formado, escaso sangrado rojo obscuro. Miembros inferiores con edema +.

Paciente delicada. Pronóstico reservado. Diagnóstico: embarazo de 38 semanas de gestación más sufrimiento fetal agudo.

Se intenta localizar al ginecoobstetra para la resolución del embarazo, el cual no contesta. Se informa a la paciente y familiar responsable de manera clara y detallada sobre la técnica quirúrgica de cirugía de urgencia cesárea tipo Kerr, los beneficios consistentes en evitar la asfixia neonatal por sufrimiento fetal, así como los riesgos inherentes como hemorragia, la cual puede llevar a la muerte, choque séptico, hemorrágico, hipovolémico, infecciones, lesiones a otros órganos, con lo cual estuvieron de acuerdo y firmaron de puño y letra el documento denominado "Carta consentimiento bajo información de procedimientos diagnósticos y terapéuticos de alto riesgo».

En la Clínica Uno, se dio inicio a la cirugía de urgencia, cesárea tipo Kerr, interviniendo como cirujano un médico diferente al imputado y éste como ayudante. A las (19:56 horas), personal de enfermería reporta a la paciente con hipotensión arterial y taquicardia. Exploración física: tensión arterial de 60/30, frecuencia cardiaca de 130 por minuto, frecuencia respiratoria 21 por minuto, temperatura de 36.5 grados centígrados. Paciente con palidez generalizada de piel y tegumentos, estuporosa, desorientada, escala de coma de Glasgow 9, precordio con taquicardia. Abdomen presenta fondo uterino por arriba de cicatriz, sangrado rojo rotulante, con útero flácido, sin tono, por lo que concluyo los diagnósticos: hemorragia obstétrica secundaria a atonía uterina. Choque hipovolémico grado III. Por lo que se inicia tratamiento con oxígeno suplementario, soluciones cristaloides 7,500 en cargo y uterotónicos, oxitocina $5 \mathrm{VI}$, IV. Misoprostol $800 \mu \mathrm{g}$. Vía rectal, carbetocina 100 microgramos IV, masaje bimanual uterino más gluconato de calcio 1 gramos IV, se canaliza por dos vías. Paciente grave, pronóstico reservado a evolución. Nuevamente se insiste en localizar a ginecoobstetra y no contesta. 
Se inicia cirugía de histerectomía total abdominal, interviniendo como cirujano otro médico y el imputado como ayudante. Al día siguiente, a las (02:00 horas) se realiza nota de traslado: sale paciente con diagnósticos de postoperada de laparotomía exploradora más histerectomía abdominal por presentar atonía uterina más choque hipovolémico hemorrágico grado III en tratamiento. Complicaciones: choque hipovolémico grado III y en tratamiento más sangrado en capa. Sangrado de 4,000 mililitros más uresis 250 mililitros. En el transquirúrgico se le administran soluciones cristaloides más sangre fresca total de 1,250 mililitros previas pruebas cruzadas. Signos vitales: TA 90/100 sistólica, 50-60 diastólica. FC 170/100. Saturación de oxígeno 95-99\%, con tratamiento a base de norepinefrina. Sale con tubo endotraqueal. La paciente requiere manejo especializado en Unidad de Cuidados Intensivos. A las (2:25 horas) se entrega a paciente en el Hospital Dos, aunque existía la urgencia y no se había anticipado el traslado, existió cierta resistencia al ingreso de la paciente, quitándonos hojas de anestesiología y corroborando la existencia de signos vitales...

3. Nota del Hospital Dos.

Se recibe en admisión de urgencias de G y 0 del Hospital, a las 03:40 a paciente en condiciones graves de Clínica Uno. Diagnóstico a su ingreso: choque hipovolémico secundario a hemorragia obstétrica por atonía uterina refractaria que ameritó histerectomía obstétrica. Se recibe paciente orointubada sin apoyo de oxígeno suplementario, con palidez de tegumentos +++, piel fría, llenado capilar retardado > 5 seg., neurológico con Glasgow de 3, sin sedación, con midriasis bilateral, sin respuesta de reflejos de estiramiento osteomuscular, refiere médico de traslado manejo con norepinefrina a $0.1 \mu \mathrm{g} / \mathrm{min}$, sin embargo, la recibimos sin aminas, no se palpan pulsos, sin embargo, hay frecuencia cardiaca, se realiza monitorización con FC de 727 lpm sin alteraciones en el D2 largo del monitor, no se sensa TA, en cuanto a lo ventilatorio con murmullo vesicular presente sin sensar saturación por oximetría de pulso, abdomen con incisión transversal con un Penrose con datos de sangrado serohemático escaso, se intentan tomar muestras de laboratorio sin poder obtener muestras, se ingresa a terapia para mejorar condiciones. A su ingreso a la $\mathrm{UCl}$ de $\mathrm{G}$ y $\mathrm{O}$, se conecta a ventiladory se inicia monitorización registrando actividad eléctrica sin pulso, por lo cual se realizan maniobras de RCP avanzado por 75 min sin respuesta, declarando muerte a las 3:20 horas.

4. Escrito de fecha 17 de diciembre de 2019, suscrito por el otro cirujano que supuestamente realizó las intervenciones quirúrgicas, del que se extrae lo siguiente:

Que ejerzo la profesión de Médico Homeópata Cirujano y Partero con cédula profesional número 0404000, expedida por la Dirección General de Profesiones de la Secretaría de Educación Pública.

5. Dictamen de necropsia de Unidad Forense del día 06 de septiembre de 2019.

El resultado fue el siguiente:

Abdomen: dos heridas quirúrgicas, la primera por cesárea de 19 centímetros, tipo Pfannenstiel, otra de 18 centímetros para laparotomía exploradora, suturadas. Abundantes coágulos y sangre en cavidad, aproximadamente 1,000 mililitros. Órganos se aprecian pálidos a los cortes. Útero: ausencia quirúrgica. Hematoma de 500 centímetros cúbicos, en toda la corredera parietocólica izquierda.

Conclusión: falleció de causa desconocida. En cuanto se tengan los resultados del laboratorio de los especímenes enviados a estudios, se ampliará la causa de muerte.

6. Peritaje de necropsia de pieza anatómica uterina, del día 21 de septiembre del 2019, de Unidad Forense, del que se extrae lo siguiente:

Conclusión. Causa de la histerectomía: inespecifica.

7. Ampliación de peritaje de necropsia de la Unidad Forense, el día 29 de octubre del 2019, del que se extrae lo siguiente: 
La causa de muerte se debió a una hipoxia anóxica encefálica, además de colapso alveolar (atelectasia multifocal), isquemia cardiaca y necrosis tubular aguda, datos compatibles con choque hipovolémico, no se reportan datos histopatológicos de atonía uterina.

8. Opinión técnica por médico en ginecología y obstetricia de Hospital Tres, 03 de enero de 2020.

Conclusiones:

a. Se realizó lo pertinente para la atención integral de la paciente a su ingreso al Servicio de la Clínica Uno, sin embargo, no se cuenta con suficientes médicos especialistas o médicos con entrenamiento quirúrgico especializado para una atención quirúrgica.

b. De lo encontrado en la cesárea, no se menciona el porcentaje de desprendimiento que la placenta presentaba, sin embargo, a pesar de los hallazgos transquirúrgicos, el recién nacido presentó un Apgar de 8/9, con un adecuado peso de 2,910 g. Mencionan que se verifica hemostasia del punto sangrante reparado en segmento medio de la histerorrafia; sin embargo, en el peritaje de la necropsia se encuentra con herida quirúrgica en el segmento inferior de 77 centímetros suturada. Esto nos habla de una prolongación de la histerotomía al momento de la cirugía, con riesgo de desgarro de las arterias uterinas y formación de hematomas en ligamentos anchos. En el peritaje de la necropsia también se menciona que en el abdomen se encuentran abundantes coágulos y sangre en cavidad aproximadamente de $1,000 \mathrm{~mL}$. Hematoma de $500 \mathrm{~cm}^{3}$ en toda la corredera parieto-cólica izquierda. Y en la ampliación, se informa que la causa de muerte se debió a datos compatibles con choque hipovolémico. No se reportan datos histopatológicos de atonía uterina.

c. Se concluye que probablemente existió una prolongación de la histerorrafia, durante la cesárea, con desgarro de la arteria uterina izquierda, con formación de hematoma en retroperitoneo extendiéndose hasta la corredera parieto-cólica ipsilateral más sangrado del segmento uterino. Que posteriormente provocó el estado de choque, por tal motivo las medidas realizadas no fueron eficaces, ya que no fue tratada la causa del choque hipovolémico.

9. Declaración de mujer testigo, del 7 de agosto del 2020:

Mujer de 44 años de ocupación técnico radiólogo flebotomista. Trabajo en laboratorio clínico. Que el cinco de septiembre del 2019, me encontraba laborando para tomar muestras en el hospital homeopático y siendo alrededor del medio día el Dr. Tratante (imputado), en forma verbal, me dio la instrucción de que requería estudio de laboratorio de las pacientes que estaban ingresando para procedimiento y que ya estaban en piso, realicé la toma de sangre de la paciente hoy occisa a quien encontré consciente en condiciones favorables, en sus cabales, sana...

Con base a todo lo anterior, hago los siguientes:

\section{Comentarios médico-legales}

Resultan evidentes las múltiples contradicciones existentes entre lo declarado por el afectado, la declaración del médico imputado y las notas del expediente clínico, sobre la atención médica proporcionada por el imputado a la finada, de las cuales resalta lo siguiente:

1. El doctor imputado afirmó en su declaración que el facultativo que efectuó la cirugía fue otro médico, siendo que, de acuerdo con lo referido en el archivo clínico, existen múltiples notas médicas, de enfermería y de anestesiología en las cuales él está como médico tratante y como cirujano, por lo que, con toda esta evidencia, es posible establecer categóricamente que el galeno que efectuó el tratamiento médicoquirúrgico, los días cinco y seis de septiembre del 2019, a la finada, fue el médico imputado.

2. Asimismo, el doctor imputado ostenta el título de Médico Homeópata Cirujano y Partero, por lo que se puede determinar que no estaba capacitado para efectuar una cesárea ni para manejar las complicaciones que se presentaron, ni reflexionó acerca de sus deberes que en su posición de Médico Homeópata Cirujano 
y Partero son de su competencia, transgrediendo lo establecido en la Ley General de Salud, que en su Artículo 81, establece: ...Para la realización de los procedimientos médicos quirúrgicos de especialidad se requiere que el especialista haya sido entrenado para la realización de los mismos en instituciones de salud oficialmente reconocidas ante las autoridades correspondientes...

a. Por lo que se podría afirmar que existió intrusismo que, de acuerdo con la literatura: Se define como «la realización de actos propios de una profesión por quien carece de la titulación académica correspondiente». Por lo que, desde un inicio, cuando supuestamente dijo que ingresó la paciente y que presuntamente presentaba sufrimiento fetal agudo, surge la pregunta: ¿por qué no la transfirió a otro hospital que contara con especialista en Gineco-Obstetricia, sabiendo que no contaría con uno en la unidad médica? Esto de acuerdo a lo determinado en la NORMA Oficial Mexicana NOM-007SSA2-2016, Para la atención de la mujer durante el embarazo, parto y puerperio, y de la persona recién nacida.

b. Sobre lo anterior, resulta conveniente hacer la siguiente reflexión: en la medida en que se trata de la responsabilidad médica, que no puede ser fundada sino en la culpa, habría que admitir, en primer lugar, que el autor del hecho u omisión culposa es responsable personalmente respecto de la víctima y, enseguida, el otro médico que ha cometido algún acto culposo, también debe ser responsable de los resultados si participó en la intervención quirúrgica de la parturienta ya que, con su contribución, modificó el archivo clínico, al efectuar notas médicas defectuosas que transgreden la Norma Oficial Mexicana «Del expediente clínico»y, con ellas, trató de evitar la responsabilidad del imputado, constituyéndose en una mala práctica médica y en un posible delito.

Respecto a lo mencionado, se ratifica en la siguiente tesis:

\section{Responsabilidad civil por negligencia médica. Su actualización en torno a los integrantes de un equipo médico. ${ }^{2}$}

3. Aunado a lo anterior, resulta obvio que la unidad hospitalaria donde se llevaron a cabo los procedimientos quirúrgicos, no cuenta con los recursos humanos, técnicos ni con los insumos a fin de atender urgencias obstétricas, de acuerdo con lo determinado en la Norma Oficial Mexicana NOM-007-SSA2-2016 «Para la atención de la mujer durante el embarazo, parto y puerperio, y de la persona recién nacida» en su numeral 5.1.8

4. En cuanto a la hemorragia que presentó la gestante y que la llevó al choque hipovolémico, resulta conveniente mencionar lo referido en la literatura médica y que, en el presente caso, evidencia que pudo deberse a la técnica quirúrgica, por lo siguiente:

La hemorragia en la cesárea se produce por razones que incluyen atonía uterina, traumatismo tisular (extensiones del ángulo uterino). El riesgo de daño vascular durante el acto quirúrgico aumenta cuando hay dificultad en la extracción del feto, ya que se puede desgarrar fácilmente la histerotomía causando un daño vascular. También la laceración de los vasos uterinos puede suceder cuando se incide el segmento inferior para la extracción de la cabeza fetal, por lo que ésta debe desimpactarse suavemente y extraerse a través de la incisión uterina. Se debe checar el útero para detectar la dextrorrotación y esto debe corregirse antes de la incisión uterina. Estas medidas reducen el riesgo de extensiones del ángulo uterino; se deben reparar esas extensiones, asegurándose de que los ángulos estén adecuadamente asegurados. ${ }^{3}$ I gualmente, las complicaciones asociadas con el parto por cesárea incluyen lesiones importantes a estructuras vasculares en el sitio de la incisión (especialmente en casos de extensión no intencionada de la incisión uterina). ${ }^{4}$ Porque una hemorragia intratable refractaria a las medidas conservadoras pueden atribuirse potencialmente a lesiones arteriales uterinas. ${ }^{5}$

Asimismo, los hematomas retroperitoneales pueden ocurrir por factores mecánicos que incluyen traumatismos por accidentes, parto $y$ 
complicaciones de las intervenciones. La causa más reconocida es la lesión de los vasos uterinos y ováricos como resultado de traumatismos o laceraciones durante la cirugía. ${ }^{6}$

Igualmente, la hemorragia postparto (HPP) constituye el 35\% del total de las muertes maternas (MM). Las principales razones son: la falta de acceso de parto asistido por personal capacitado y la práctica inadecuada o limitada del manejo activo del alumbramiento. Cuando la paciente es asistida en los servicios de salud, la demora en el reconocimiento de la hipovolemia y el inadecuado reemplazo del volumen, son la razón principal de MM.?

5. Resulta importante resaltar que, regularmente, las lesiones vasculares iatrogénicas no se documentan en los expedientes, como en el presente caso; porque, cuando ocurren, se dan justificaciones que pretenden atribuir a otras condiciones el origen del problema (como en el caso presente, en que se atribuyó la hemorragia a atonía uterina); del que es viable afirmar dos posibilidades: la primera, que fue un error diagnóstico, o la segunda, que fue determinado para evitar que se conociera la causa real derivada de una deficiente técnica quirúrgica, puesto que en el dictamen de ampliación de peritaje de necropsia de la Unidad Forense, el día 29 de octubre del 2019, sobre el útero se determina que: no se reportan datos histopatológicos de atonía uterina. La reflexión es: prestar atención a la fisiopatología de la complicación y conocer los procedimientos quirúrgicos para tratarla y describir con transparencia, en el expediente clínico, el estado del paciente.

a. Se observa que, en el manejo de la hemorragia postparto, no hubo una actuación inmediata y secuencial. Porque las principales complicaciones son por maniobras inefectivas para detener el sangrado. Ya que es innegable que la muerte de la paciente fue derivada de una transfusión sanguínea tardía, porque en esa unidad médica no contaban con banco de sangre.

Lo anterior se ratifica por lo determinado en la nota médica efectuada por el médico imputado del cinco de septiembre a las 22:00: Viendo la urgencia de que el paciente requería sangre se le piden al familiar paquetes globulares y el esposo se niega rotundamente, a pesar de que se le da la dirección, aludiendo no tener dinero, causa por la cual se les pide apoyo a alumnos de medicina, obteniendo la sangre y cubriendo la urgencia de momento y siguiendo protocolo.

Sin mencionarse si se cumplió con lo establecido en la NORMA Oficial Mexicana NOM-253SSA1-2012, Para la disposición de sangre humana y sus componentes con fines terapéuticos.

6. Con relación a las Cartas de consentimiento informado que se encuentran en el expediente:

Hay una hoja de autorización y registro de intervención quirúrgica del cinco de septiembre del 2019, sin hora y sin firma de autorización del paciente; una hoja de conformidad del paciente y familiares para tratamiento médico y/o quirúrgico del cinco de septiembre del 2019, en el que aparece como médico tratante el imputado y en el texto aparece otro doctor (letra casi ilegible que al parecer corresponde a otro cirujano) al que supuestamente se autorizan los tratamientos, sin especificar qué tratamientos y sin firma de testigos; cartas de consentimiento informado sin firmas de testigos; sobre este último punto, es importante señalar que sí tiene importancia relevante, ya que el denunciante expresa que existieron alteraciones en el contenido del consentimiento informado de dicho expediente, además de que sí influye esta situación en los resultados del tratamiento médico quirúrgico efectuado a la finada y en la determinación de la mala práctica médica llevada a cabo, puesto que se trató de afirmar que fue otro doctor quien efectuó los tratamientos quirúrgicos, cuando existe evidencia que el cirujano fue el imputado.

Por lo que, No se elaboraron como lo establece la NORMA Oficial Mexicana NOM-004-SSA3-2012, Del expediente clínico.

7. La estructura del expediente clínico de este caso no cumple con lo establecido en la NOM-004, porque: existen notas médicas que no tienen fecha ni hora, sin nombre y firma de quién la realiza y algunas con letra ilegible. 


\section{Conclusiones médico-legales}

1. De acuerdo con toda la evidencia, que se encuentra en el expediente clínico, es posible establecer categóricamente que el doctor que efectuó el tratamiento médico-quirúrgico a la finada fue el facultativo imputado, por lo tanto, es factible determinar que incurrió en mala práctica médica, por lo siguiente:

a. Haber recabado el consentimiento informado para la operación cesárea sin la firma de testigos.

b. Haber realizado una operación cesárea a la fallecida, sin contar con la cédula de especialidad en ginecología y obstetricia y sin la competencia para hacerlo;

c. Ocasionar una lesión en la arteria uterina por una prolongación de la histerotomía, que ocasionó una hemorragia de la cual no se percató, de lo que derivó un choque hipovolémico, complicaciones que no supo ni pudo controlar dando como resultado la pérdida de la vida de la paciente, de acuerdo con el reporte forense.

d. Transgredir la Norma Oficial Mexicana NOM253-SSA1-2012 «Para la disposición de sangre humana y sus componentes con fines terapéuticos»;

e. Haber ejecutado tratamientos médicoquirúrgicos a la paciente en una unidad hospitalaria que no cuenta con los recursos humanos, técnicos ni con los insumos a fin de atender urgencias obstétricas, de acuerdo con lo determinado en la Norma Oficial Mexicana NOM-007-SSA2-2016 «Para la atención de la mujer durante el embarazo, parto y puerperio, y de la persona recién nacida».

f. Haber efectuado notas médicas en el expediente clínico en las cuales se observan contradicciones e irregularidades $\mathrm{y}$, tener notas de un médico que no intervino quirúrgicamente a la paciente; transgrediendo lo establecido en la Norma Oficial Mexicana NOM-004-SSA3-2012 «Del archivo clínico», situación que sí afecta el estudio del hecho, puesto que es evidente que trataron de ocultar la responsabilidad del doctor imputado. g. En consecuencia, existió mala práctica médica por parte del médico que supuestamente realizó las intervenciones quirúrgicas, ya que, con su participación, alteró el expediente clínico.

h. Existe contradicción en lo declarado por el doctor imputado y lo descrito en sus anotaciones médicas y hojas de consentimiento informado, con la declaración efectuada por el denunciante, la flebotomista del laboratorio particular y lo referido en las notas y hojas de anestesia, por lo que se puede determinar que: tanto lo descrito en su declaración, como lo documentado en el archivo clínico, por parte del médico imputado, es una falacia.

\section{DISCUSIÓN}

La mala práctica médica es un tema complejo, en gran parte debido a que sus efectos son de gran alcance. Se caracteriza porque quien proporcionó la atención actuó en forma negligente, imprudente o con impericia, implicando que esta persona no era competente o razonablemente hábil, perjudicando al paciente incluso, hasta provocar su deceso, sin embargo, existen casos en que el médico actúa de tal manera que sus acciones más que calificarlas de negligentes hacen dudar acerca de la intencionalidad, porque se actúa a sabiendas de que lo que está haciendo es ilegítimo y, a pesar de ello, lo lleva a cabo, configurándose una acción francamente dolosa, tal fue el caso que se acaba de analizar y se deja al criterio del lector este razonamiento para la reflexión.

El expediente clínico es una de las herramientas más esenciales en la defensa por presunta mala práctica médica; es un documento legal que se utiliza buscando demostrar el proceso de pensamiento que conduce al diagnóstico y opciones de tratamiento. Todo lo relacionado con el manejo del paciente debe registrarse.

Un expediente clínico bien documentado sirve a dos propósitos valiosos: 1) refleja el historial del paciente y la atención médica correspondiente, lo que permite la continuidad del cuidado; y 2) puede proporcionar una defensa para un médico que enfrenta acusaciones por mala práctica médica, al detallar la adhesión a los estándares de cuidado. 
La documentación clínica necesita el esfuerzo concertado de varias personas involucradas en la atención al paciente; dicho sucintamente, todos los que brindan atención a un paciente son responsables de documentar la atención y las recomendaciones que se han proporcionado y articulado al paciente. Sin embargo, el doctor es la persona que debe supervisar este proceso y es el principal responsable del historial del paciente.

Un médico podría no darse cuenta de las deficiencias en su documentación hasta que se enfrente a una denuncia por mala práctica médica o una mala conducta profesional, y es natural que se sienta nervioso y un poco en pánico cuando es demandado por mala práctica médica. En esos momentos puede haber una fuerte tentación de agregar a la historia clínica para «aclarar» qué realmente ocurrió, o eliminar información potencialmente perjudicial, borrar o eliminar notas médicas o notas de enfermería que entran en conflicto con su defensa; además, si el abogado del demandado sospecha que el expediente contiene información contraria a su teoría del caso, podría aconsejar a su cliente que lo altere o que, incluso, elabore un expediente completamente nuevo pero falso, y también a estructurar una declaración impecable pero llena de mentiras. No obstante, contrario a las intenciones del abogado, a menudo la versión de los eventos del paciente entra en conflicto con lo que el médico ha documentado y, en tales casos, se pone en evidencia que el facultativo ha mentido, como sucedió en el caso estudiado.

Por otro lado, la documentación de los hechos que respaldan al médico en un juicio ofrecerá protección, al demostrar que el estándar de cuidado fue seguido. Cualquier cambio en el expediente clínico se puede descubrir fácilmente durante el curso de la investigación previa al juicio. Los peritos de la parte contraria son capaces de detectar cambios a los expedientes, contrastando la declaración del denunciante o del paciente con la información de las anotaciones médicas. En un hospital, podría haber varios facultativos o enfermeras haciendo anotaciones en el expediente, por lo que es posible resaltar una inconsistencia si está modificado. Por ejemplo, una versión anterior conflictiva del expediente puede haber sido enviada a otros médicos o instituciones y quedará en evidencia cuando los peritos médicos que evalúan el caso comiencen a reunir el expediente clínico del paciente, que se encuentra que fue enviado o dejado con otro doctor o en otra institución donde fue trasladado el paciente con el propósito de continuar su tratamiento o para un estudio; situación que sucedió en el caso estudiado, ya que el médico imputado dejó las hojas de anestesia, en el Hospital Dos junto con la paciente y, con ellas, se demostró que tanto su declaración, como el archivo clínico que entregó a la autoridad no establecían los hechos.

Expedientes clínicos, claramente falsos, podrían ser utilizados por un demandante para arrojar dudas sobre la calidad de atención que recibió el paciente. Una prueba de alteración del expediente sin una buena causa y adecuada autenticación, tiene graves consecuencias en un juicio por responsabilidad profesional. Modificar un expediente clínico implica alterar la evidencia. Dicha prueba destruirá la credibilidad del acusado ante un juez y dejará la fuerte impresión de que él o ella están tratando de ocultar la verdad.

En otro orden de ideas, se demostró que el doctor imputado siempre fue el médico tratante y el que efectuó los procedimientos quirúrgicos, por lo tanto, fue responsable de los resultados.

En este mismo sentido, en la literatura médica se describe lo siguiente: el contexto científico Harrison define al médico tratante como la persona responsable del paciente y su enfermedad, pues es aquel que se hace cargo del caso y guía al paciente en el transcurso de la enfermedad. Por tanto puede asumirse que el médico tratante es el líder de un equipo clínico multidisciplinario que conoce integralmente la situación del paciente y se responsabiliza de las decisiones relacionadas con el diagnóstico y tratamiento, es decir, podría denominarse como el médico de cabecera. ${ }^{8}$

A pesar de que los médicos tratantes tienen la responsabilidad general de los pacientes bajo su cuidado, en muchos casos ellos delegan tareas a otros profesionales de la salud, pero tienen la obligación ética y profesional de garantizar que los médicos a los cuales les delega la continuidad en el manejo del paciente tengan la capacidad de realizar las tareas de la atención médica con estándar apropiado, y de obtener el consentimiento, que es imprescindible.

Al delegar la atención de los pacientes, se debe recordar que la persona que asume la 
responsabilidad de la atención médica continuada de los pacientes no tiene la misma profundidad de comprensión sobre sus afecciones médicas que el prestador de atención médica que le está delegando la responsabilidad del manejo del paciente. La transferencia se debe documentar, verbalmente y en el expediente clínico. Un ejemplo podría incluir el presente caso, donde el facultativo tratante, que es un médico general, delega la responsabilidad a otro médico general. Cuando un profesional de la salud delega la prestación de servicios de salud a otro profesional de la salud, el delegado aplica los principios del consentimiento informado antes de proporcionar la atención médica informando al paciente y/o sus familiares que él no cuenta con la especialidad para efectuar al acto médico-quirúrgico. Cuando un profesional de la salud debe proporcionar o realizar una forma específica de atención médica, la tarea de informar a un paciente sobre los riesgos materiales y, sobre todo, si el tratamiento involucra algún acto determinado en la norma sobre el consentimiento informado, éste debe ser recabado por el paciente para que acepte que ese otro cirujano realice el procedimiento de diagnóstico o tratamiento. El registro debe documentar claramente quién asume la responsabilidad de la atención médica, incluso horarios de inicio y finalización con fechas específicas. Identificar quién es el facultativo responsable del paciente y el encargado de seguir el tratamiento y sus resultados. Todas las acciones de seguimiento deben documentar lo que fue hecho, por quién y cuándo.

En cuanto a la delegación de actos médicos en general, una persona autorizada para practicar medicina tendrá la autoridad de delegar a cualquier doctor calificado y debidamente capacitado cualquier acto médico que un doctor razonable y prudente encontraría dentro del alcance del juicio médico sólido para delegar si, en opinión del cirujano delegante, el acto puede ser adecuado, seguro y realizado por la persona a quien se delega el tratamiento y el acto se realiza de la manera habitual, no en violación de ningún principio o norma que rigen la práctica de la medicina. El médico delegante compartirá la responsabilidad solidaria de los actos médicos del doctor que realiza los actos delegados. Por lo que es necesariamente ético y profesional que un médico tratante que requiera delegar la continuidad del manejo de su paciente a otro galeno, debe cerciorarse de que ese otro doctor cuente con la especialidad y la capacidad de brindar un manejo óptimo al paciente y el médico al que se le pretende delegar el seguimiento del paciente, también debe actuar honestamente y decidir si cuenta con los conocimiento y capacidad para hacerlo, de lo contrario negarse a ello; respetar la decisión y a poyar a un médico delegado que indica que no tiene los conocimientos y habilidades suficientes o experiencia para emprender la tarea es importante, porque un profesional de la salud delegado reconoce y trabaja dentro de los límites de su competencia profesional y alcance definido de la práctica, realiza la tarea para cumplir con sus responsabilidades legales y profesionales y sobre todo, informa al paciente y/o familiares acerca de quién es y porqué va a participar en su manejo, con la meta de obtener un consentimiento informado válido, asegurando que se complete el documento de consentimiento.

El razonamiento anterior reconoce que los doctores podrían legalmente delegar actos médicos de emergencias a facultativos debidamente capacitados y calificados. Cuando un médico desea delegar el manejo del paciente a otro doctor no especialista, para realizar un acto médico, debe estar consciente de que ese tratamiento, realizado por doctores no capacitados o especializados, lo más probable es que no se ajuste al estándar de atención que se aplicaría si un cirujano especialista llevara a cabo el acto, por lo que, tanto él, como el doctor tratante y el médico al que le delega su manejo, son responsables de los resultados que se obtengan.

Igualmente, se tiene que tomar en cuenta también que las consecuencias lesivas pueden ser debidas no sólo a la actuación de un determinado profesional, sino a que a la aparición de las mismas ha podido contribuir la actuación de varios individuos, por ello es muy importante que en el expediente clínico se pueda identificar perfectamente al facultativo que realizó cada uno de los actos médicos, con la finalidad de prevenir un gran número de estas acciones y evitar la tendencia creciente que le quitará al doctor toda voluntad de asumir responsabilidades, impulsándolo a pasarle la «carga» a otro, «para evitar o para salvar la responsabilidad», como se pretendía en el hecho estudiado. 
Por consiguiente, en estos casos el médico delegante junto con el doctor que realiza el acto sin estar capacitado, serán responsables solidarios, acorde a lo establecido en el Código Civil Federal, en el Artículo 1917.- «Las personas que han causado en común un daño, son responsables solidariamente hacia la víctima por la reparación a que están obligadas de acuerdo con las disposiciones de este Capítulo».

En cuanto al consentimiento informado, es simultáneamente requisito legal y ético de los actos jurídicos de la relación médico-paciente, siendo una institución primordialmente jurídica con profundas implicaciones bioéticas. ${ }^{9}$ Y deberá ser recabado por el profesional de la salud que indica el procedimiento o por el que vaya a practicarlo, según lo determine el respectivo servicio de salud, priorizando el derecho del paciente a la mejor calidad de la información». ${ }^{10}$

Y se confirma por lo determinado en la siguiente tesis:

\section{Consentimiento informado. Derecho fundamental de los pacientes:"1}

El consentimiento informado es consecuencia necesaria o explicitación de derechos a la vida, a la integridad física y a la libertad de conciencia, el cual consiste en el derecho del paciente de otorgar o no su consentimiento válidamente informado en la realización de tratamientos o procedimientos médicos. En tal sentido, para que se pueda intervenir al paciente, es necesario que se le den a conocer las características del procedimiento médico, así como los riesgos que implica tal intervención. A través de éste el paciente asume los riesgos y consecuencias inherentes o asociados a la intervención autorizada; pero no excluye la responsabilidad médica cuando exista una actuación negligente de los médicos o instituciones de salud involucrados.

Sin embargo, en el caso estudiado existieron una serie de anormalidades en la integración de los documentos de consentimiento informado, situación que, además de no cumplir con la normatividad, se puede determinar que fue elaborada tratando de evitar una posible denuncia por mala práctica médica, a pesar de ello, los que los integraron no actuaron negligentemente y es factible, incluso, decir que lo hicieron dolosamente, situación que se aparta completamente de los principios éticos que regulan el ejercicio de la medicina.

Sobre lo anterior, en la literatura médica de México, ${ }^{12}$ se encuentra referido lo siguiente:

"Uno de cada tres expedientes no tenía el Cl (consentimiento informado) y aún más, 20\% de los que afirmaron haber signado el documento, éste no fue encontrado, así mismo y más grave aun, 13\% de casos referidos como no firmados, contenía un documento con firmas apócrifas, no identificadas. El 63\% de los expedientes tenían el Cl, de éstos, llama la atención un alto número de documentos no signados que no corresponden, lo que orienta a falsificación de los mismos. De 30 expedientes que se signaron y no fueron encontrados, existe la posibilidad de que el informante haya mentido o se haya sustraído el documento. Aún más alarmante fue encontrar 78 formatos firmados cuando los pacientes o familiares no lo hicieron, lo que se traduciría en una falsificación del documento».

\section{CONCLUSIÓN}

La mala práctica médica es un tema complejo, en gran parte debido a que sus consecuencias son de gran alcance por sus efectos negativos en la salud y la vida de los pacientes; no sólo involucra las acciones que se llevan a cabo en el cuerpo y/o mente del paciente sino que, de igual forma, engloba toda la documentación que se elabora e integra sobre el manejo del paciente y que se debe cuidar y mantener intacta en un expediente clínico. La estructuración correcta de los expedientes clínicos puede dar lugar a la disminución de las reclamaciones médicas; es muy importante que el médico tratante documente y supervise su integración correcta, esta será la única forma para que demuestre que el tratamiento se realizó adecuadamente. Es desalentador observar que, a pesar de saber la importancia de mantener un expediente adecuado, todavía se observan deficiencias o actos ilegítimos en su integración.

Se debe insistir en que este documento será de gran ayuda para el médico acusado de mala práctica médica, porque siempre será un foco principal 
de escrutinio en una denuncia o demanda penal o civil e, igualmente, la alteración de un expediente clínico puede afectar negativamente la credibilidad de los profesionales que lo integraron. Incluso una corrección o adición hecha correctamente podría, ante un mal resultado, poner en duda la credibilidad de todo el expediente. Un expediente alterado será más que una desventaja en defensa de un caso, además de que es viable descubrir fácilmente durante el curso de la investigación previo al juicio. Los abogados de los demandantes son conscientes de la tremenda ventaja que pueden obtener si ha habido una alteración subrepticia del historial médico del paciente. Saben que no hay nada mejor que un historial médico alterado para convertir un caso de descarte, o incluso un posible perdedor, en un ganador. Igualmente, los expedientes inexistentes significan que no hay defensa.

En otro orden de ideas, la buena práctica clínica y el consentimiento informado son inseparables. El respeto a la autonomía del paciente en la práctica clínica es de gran importancia moral en la sociedad. La moral y responsabilidad legal de obtener el consentimiento informado médico depende de la transmisión de información apropiada al paciente. La documentación clínica debe reflejar esta toma de decisiones informada entre el prestador de servicios de atención médica y el paciente.

Las decisiones de los pacientes deben hacerse independientemente y basadas en el espectro completo de conocimiento de su enfermedad y no realizando documentos de consentimiento informado que no son acordes a la norma, o que sean falsos porque no fueron suscritos por el paciente, el familiar responsable o por testigos; o hacerlos simplemente con la idea de a parentar haber cumplido con la normatividad y así tratar de ocultar evitar un procedimiento legal por no estar integrado al expediente clínico y que ello haya influido en malos resultados en el manejo del paciente.

Los médicos pueden legalmente delegar actos médicos de emergencias a otros médicos debidamente capacitados y calificados. Un tratante que desea delegar el manejo del paciente para realizar un acto médico a otro galeno no especialista, debe estar entendido de que un tratamiento realizado por médicos no capacitados o especializados, lo más previsible es que no se apegue al estándar de atención que se daría si un cirujano especialista realizara el acto, por lo que, tanto él, como médico tratante y aquel al que le delega su manejo, son responsables de los resultados que se obtengan.

El perder el prestigio, por deshonestidad en el ejercicio de la medicina, representa la decadencia de esta carrera. Sin embargo, por valiosa que sea para los pacientes y los doctores, la honestidad no ha sido una preocupación importante en la ética médica ni un principio valioso para los galenos. Podría pensarse que es una exageración aseverar que la honestidad no se inculca en las escuelas y facultades de medicina, ni se reconoce en el conjunto de conocimientos médicos, pero no es producto de la imaginación, porque se observa en el caso que se analizó.

\section{REFERENCIAS}

1. Ramos Ramos A, Beas Nava JA, Suro Cedano G, Vázquez Cortés FJ, Machuca Martínez LF, De Alba Macías P et al. El conflicto en la práctica profesional de atención a la salud. México: Universidad de Guadalajara; 2008. pp. 276, 278, 281, 282.

2. Gaceta del Semanario Judicial de la Federación. Décima época. Num. Registro: 2015868. Tribunales Colegiados de Circuito. Tesis aislada. Libro 49, Diciembre 2017, Tomo IV. Materia Civil. Tesis 1.4 ‥ . 58. Pág. 2261.

3. Field A, Haloob R. Complications of caesarean section. The Obstetrician \& Gynaecologist. 2016; 18 (4): 265-272.

4. Asicioglu O, Gungorduk K, Asicioglu BB, Yildirim C, Gungorduk OC, Ark C. Unintended extension of the lower segment uterine incision at cesarean delivery: a randomized comparison of sharp versus blunt techniques. Am J Perinatol. 2014; 31 (10): 837-844.

5. Kwon JH, Kim CS. Obstetric iatrogenic arterial injuries of the uterus: diagnosis with US and treatment with transcatheter arterial embolization. Radiographics. 2002; 22 (1): 35-46.

6. Rafi J, Khalil H. Maternal morbidity and mortality associated with retroperitoneal haematomas in pregnancy. JRSM Open. 2018; 9 (1): 1-9.

7. Asturizaga P, Toledo Jaldin L. Hemorragia obstétrica. Rev Med La Paz. 2014; 20 (2): 57-68.

8. Rojas Giraldo EM, Aristizábal Agudelo L, Babilonia Negrete $\checkmark$. Alcance del concepto del médico tratante en las acciones de tutela para proteger el derecho al diagnóstico. Revista CES Derecho. 2014; 5 (2): 197-219.

9. Vázquez GAR, Ramírez BÉJ, Vázquez RJA, Cota GF, Gutiérrez MJA. Consentimiento informado. ¿Requisito legal o ético? Cir Gen. 2017; 39 (3): 175-182.

10. Berro Rovira G. Consentimiento informado. Rev Urug Cardiol. 2013; 28: 17-31. 
11. Décima Época. Núm. de Registro: 2001271. Primera Sala. Semanario Judicial de la Federación y su Gaceta. Libro XI, Agosto de 2012, Tomo 1. Materia(s): Constitucional. 1 . XLIII/2012 (10a.). Página 478.
12. Vizcaya BDM, Zúñiga VFA, Pérez CP, Cobos AH. Conocimiento de los pacientes sobre el consentimiento informado en un hospital general. Rev Fac Med UNAM. 2014; 57 (5): 5-13. 